\title{
Correlation of Contrast-Enhanced Ultrasonographic Features with Microvessel Density in Papillary Thyroid Carcinomas
}

\author{
Qi Zhou ${ }^{\& *}$, Jue Jiang ${ }^{\star}$, Xu Shang, Hong-Li Zhang, Wen-Qi Ma, Yong-Bo Xu, \\ Hua Wang, Miao Li
}

\begin{abstract}
Background: The purpose of this study was to investigate the correlation of contrast-enhanced ultrasonographic (CEUS) features with microvessel density (MVD) in papillary thyroid carcinomas (PTCs). Materials and Methods: Contrast-enhanced ultrasonography (CEUS) was performed in 62 patients (17 men and 45 women) with PTC. Tomtec software was applied to analyze the time intensity curve of CEUS. Immunohistochemistry was performed to evaluate the level of MVD in papillary thyroid carcinoma. Then the relationship between quantitative feature and the level of MVD was analyzed using SPSS 16.0 software. Results: The mean peak intensity of PTC tissues was lower than that of peripheral thyroid parenchyma $(61.9 \pm 11.8 \%$ vs $100 \%, p<0.05)$. The MVDs of CD34 and CD31 antibodies staining were 38.0 \pm 6.1 and 37.9 \pm 5.1 respectively in $62 \mathrm{PTC}$ samples. A significantly positive correlation was observed between peak intensity and MVD in PTC tissues $\left(P_{\mathrm{CD} 34}<0.01\right.$, $\left.r_{\mathrm{CD} 34}=0.838, P_{\mathrm{CD} 31}<0.01, \mathrm{r}_{\mathrm{CD} 31}=0.837\right)$. Conclusions: The peak intensity in CEUS could reflect the MVD in PTC tissues. Therefore, quantification of CEUS seems to be helpful for assessment of MVD in PTC tissues.
\end{abstract}

Keywords: Contrast-enhanced ultrasonography - microvessel density - papillary thyroid carcinoma

Asian Pac J Cancer Prev, 15 (17), 7449-7452

\section{Introduction}

Thyroid carcinomas are the most common tumor of endocrine organs, and papillary thyroid carcinomas (PTC) account for $70 \%$ to $80 \%$ of thyroid carcinomas (Asioli et al., 2010). PTC is well-differentiated thyroid carcinomas that usually have a good prognosis, and only a few patients with PTC develop recurrence or metastases to other organs, and the cancer death of PTC is relatively low. However, PTC is associated with many aggressive tumors (less than $1 \%$ of all thyroid nodules), such as tall cell, columnar cell, or diffuse sclerosing variants of the PTC (Santacroce et al., 2009; AsioliErickson et al., 2010).

Angiogenesis, the process of new blood vessels development, is a critical step for growth, local extension and metastasis of varieties tumor cells such as hepatocellular carcinoma, thyroid carcinoma, lung cancer, breast cancer, melanoma and renal cell carcinoma (Yang et al., 2002; Wang et al., 2007). Microvessel density (MVD), a measure of tumor angiogenesis, has considered to be an important reference standard in the quantification of blood vessels and for prediction of the likelihood of tumor metastases (Liotta et al., 1974; Du et al., 2008). However, MVD counting using surgical and biopsy specimens are invasive and prone to sampling errors and may not be ideal tools for clinical purposes (Du et al., 2008). Thus, it is essential to establish an in vivo assay for estimating tumor vessels that is noninvasive, able to sample the entire tumor, and repeatable at frequent intervals. Currently, several studies report contrast-enhanced ultrasonography (CEUS) technique can be applied to detect microvessels and quantitatively assess the vascular features of tumors such as solid renal tumors (Tamai et al., 2005), liver tumors (Solbiati et al., 2004) and prostate carcinoma (Halpern et al., 2005). It can visualize the microcirculation of tumor and offer information on tumor vascularity in detail (Hayashi et al., 2002; Schlemmer et al., 2004; Wang et al., 2005). Quantitative feature of CEUS are assessed with time-intensity curves, and this method can be applied to evaluate tumor angiogenesis in vivo (Bartolotta et al., 2006; Zhuang et al., 2012). Thus, CEUS can be recognized as a promising and powerful tool for evaluation of papillary thyroid carcinoma angiogenesis.

The relationship between CEUS feature and MVD in PTC is vital but only a littlereporthas been made until now. Whether CEUS can reflect tumor vascularity in PTC is still unclear. The aim of this study was to clarify the correlation of CEUS with MVD in PTC and to provide noninvasive indices for clinical tumor vessel evaluation. Thus, we investigated the association between peak intensity (PI) 
of tumors on CEUS and MVD values in this study.

\section{Materials and Methods}

\section{Study population}

Sixty-two patients (17 men and 45 women; range, 22-61 years; mean age, $42.5 \pm 6.3$ years) were recruited into this study from February 2010 to May 2012. In order to ensure the accuracy of the same nodules, multiple nodules as well as single nodules will be conducted for intraoperative ultrasonography and positioning. The diameters of the nodules ranged from 5.1 to $23.1 \mathrm{~mm}$ (average, $8.3 \pm 3.7 \mathrm{~mm}$ ). The study was approved by the local Ethics Committee, and informed consent was obtained from all patients.

\section{CEUS evaluation}

CEUS was prepared for the patients need for surgery. CEUS was performed with Siemens Acuson Sequoia 512 color Doppler ultrasonic diagnostic apparatus and a $15 \mathrm{~L} 8 \mathrm{~W}$ linear array probe with transmitting frequency of 7MHz.The mechanical index of CEUS was 0.32 . The contrast media (SonoVue, white freeze-dried powder) was purchased from Bracco. The SonoVuewas dissolved in $5 \mathrm{~mL}$ normal saline and after shaking by hand, $2.4 \mathrm{ml}$ was used for quick infusion into the median cubital vein. Then, $5 \mathrm{ml}$ of normal saline was immediately used as a flush. The PTC nodules were scanned firstly by conventional twodimensional ultrasound to obtain the best imaging plane. Before the injection of contrast media, the contrast presets on the machine were optimized. Then the ultrasound mode was switched on, and the key time and dynamic storage were chosen. The nodules were continuously scanned after injection of contrast media. Scanning was stopped for $2 \mathrm{~min}$. The imaging process was recorded continuously on the hardware of the ultrasonography machine. The maximum diameter of each lesion was measured. Color and power Doppler ultrasonography was performed to evaluate the intralesional vascularity, and the plane with the richest vasculature was selected for CEUS.

\section{CEUS imaging analysis}

The dynamic images were reviewed for the enhancement pattern and the echogenicity change of PTC nodules and the peripheral thyroid parenchyma. Then the TomTec software which could draw time-intensity curve quantitative was started. Tom Tec software has the motion compensation mode (compensate for respiratory motion) which can be used for the de-machine analysis, it reflects the results accurately. The regions of interest (ROIs) were selected to be in the most enhanced area within the PTC nodules and the peripheral thyroid parenchyma. The timeintensity curves were assayed for the following indices: arrival time (AT, in seconds), defined as the first point of the curve clearly above the baseline intensity followed by a further rise; peak intensity (PI), defined as the increase in signal intensity, from baseline signal intensity to maximal signal intensity measured in the selected ROI during the selected period of enhancement. The PI of peripheral thyroid parenchyma was defined as $100 \%$; time to peak (TTP, in seconds), defined as the time from the start of the injection to the maximum intensity of the curve; mean transit time (MTT, in seconds), defined as the time from the start of the rising curve to PI drop to $50 \%$.

\section{Immunohistochemical analysis}

Tumor specimens, embedded in paraffin, were analyzed by the standard immunohistochemical method to measure the MVD. Serial sections (4- $\mu \mathrm{m}$ in thickness) were selected for staining with CD31 and CD34 antibodies (FuZhouMaixin Biotechnology Company) by 2 pathologists with rich clinical experience. Microvessels labeled with the CD31 and CD34 antibody were quantified by the Weidner et al counting procedure(Weidner, 1998). Microvessel counts were evaluated by 2 pathologists with knowledge of the CEUS. Five separate areas containing the greatest number of microvessels were chosen under a low magnification $(\times 100)$, and then the microvessel numbers were counted under a high magnification $(\times 400)$. Any endothelial cell or a cell cluster that was stained in brown was considered positive. The endothelial cell at different positions which may originate in the same vessel was also considered a single countable microvessel. We did not count the microvesselwhose lumen was greater than eight of erythrocyte diameter, the microvessel with thick muscle layer or hardening area, as well as the mirovessel in area of rare tumor cells and close to normal tissue. The MVD value of the tumor was defined as the mean value of the microvessel number. Each specimen was examined 3 different times, the results error being $<5 \%$.

\section{Statistical Analysis}

All data were described as mean \pm SD. The comparison of quantitative feature in CEUS and in the MVD values between thyroid papillary carcinoma and peripheral parenchyma was analyzed by the Student t test. The correlation of quantitative feature with MVD was analyzed by the Pearson correlation analysis. $p<0.05$ was considered statistically significant. All analysis was performed with SPSS 16.0 software.

\section{Results}

Sixty PTC nodules showed low and heterogeneous enhancement compared with peripheral thyroid parenchyma (Figure 1), and the other 2 lesions showed slightly high enhancement. As shown in Table 1, the PI of CEUS the in PTC and peripheral parenchyma were $30.1 \pm 11.5 \%$ and $100 \%$, the MTT of CEUS in PTC and peripheral parenchyma were $30.1 \pm 11.5$ and $27.8 \pm 8.2$,

Table 1. Comparision of Quantitative Feature in Thyroid Papillary Carcinoma and Peripheral Parenchyma

\begin{tabular}{|c|c|c|c|c|}
\hline Position & Arrive time $(\mathrm{AT})(\mathrm{s})$ & Time to peak(TTP)(s) & Mean transit time(MTT)(s) & Peak intensity $(\mathrm{PI})(\%)$ \\
\hline thyroid papillary carcinoma & $61.9 \pm 11.8$ & $13.5 \pm 2.7$ & $18.7 \pm 3.4$ & $30.1 \pm 11.5$ \\
\hline peripheral parenchyma & $13.4 \pm 2.9$ & $18.2 \pm 3.6$ & $27.8 \pm 8.2$ & 100 \\
\hline$p$ value & 0.782 & 0.296 & 0.041 & $<0.050$ \\
\hline
\end{tabular}


and the differences of the MTT and PI in PTC nodules and peripheral thyroid parenchyma were significant $(p$ $<0.05)$, while the differences of AT and TTP were not significant $(p>0.05)$.

The mean MVD value of 62 PTCs obtained by immumohistochemical staining of CD34 and CD31 were $38.0 \pm 6.1$ and $37.9 \pm 5.1$ respectively. A significantly positive correlation was observed between PI of CEUS and MVD in PTC nodules $\left(\mathrm{p}_{\mathrm{CD} 34}<0.010, \mathrm{r}_{\mathrm{CD} 34}=0.838\right.$, $\left.\mathrm{p}_{\mathrm{CD} 31}<0.010, \mathrm{r}_{\mathrm{CD} 31}=0.837\right)$. The relationship between PI of CEUS and MVD is shown in Figure 2.

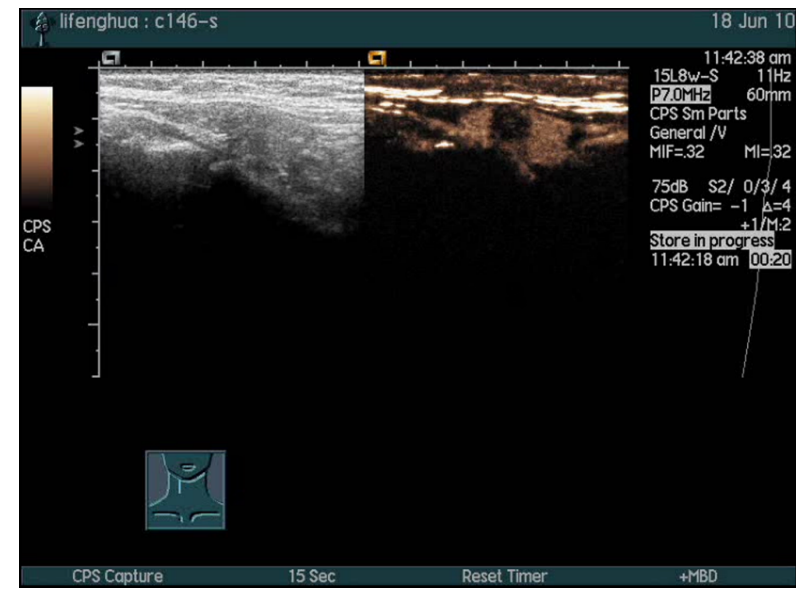

Figure 1. Contrast-Enhanced Ultrasonography of Thyroid Papillary Carcinoma

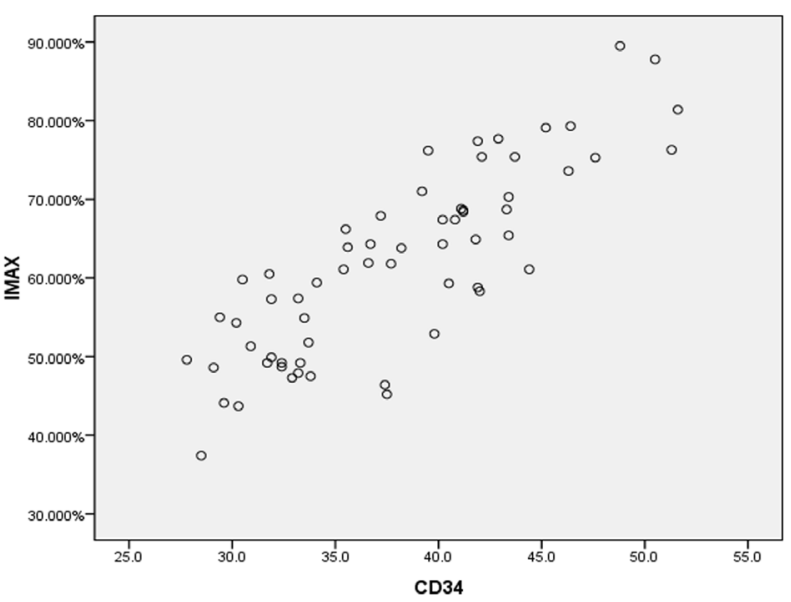

Figure 2. The Correlation between PI of ContrastEnhanced PDUS and MVD $\left(r_{\mathrm{CD} 34}=0.838, r_{\mathrm{CD} 31}=0.837\right)$

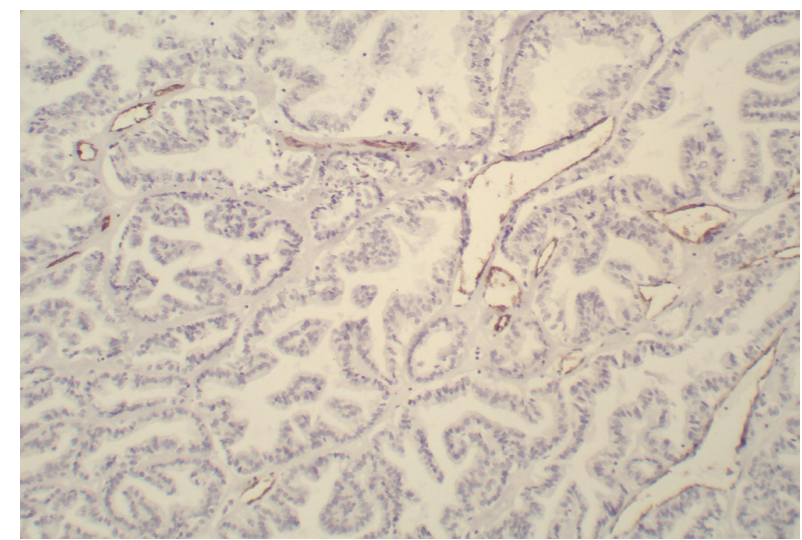

Figure 3. Microvessel Staining of PTC (CD34 Antibody, Original Magnification $\times \mathbf{1 0 0}$ )

\section{Discussion}

As one kind of thyroid carcinoma, the benign lesions of PTC is common, and the early detection and treatment of tumor can generally contribute to the good prognosis of PTC (Wang et al., 2013). The early clinical symptom of thyroid carcinomas is hidden and uneasy to be found. Many thyroid carcinomas are less than $1 \mathrm{~cm}$ in diameter and non-palpable (Brander et al., 1991; Accurso et al., 2005). Ultrasonic is a common imaging examination screening of thyroid carcinomas, because of its convenient, economy, and noninvasive features. Conventional ultrasonography and real time ultrasound elastography have been successfully used in the diagnosis of mimicking PTC (Wu et al., 2013). CEUS has been a useful screening tool for detecting changes in vascular features, such as vessel diameter, flow velocity, volume of flow, and morphologic characteristics (Kabakci et al., 2005). The contrast agent of CEUS can facilitate continuous and dynamic observation the perfusion of tumor vessels. The mean diameter of contrast agent microbubbles is only $2.5 \mu \mathrm{m}$, which makes observation of microcirculation possible (Quaia et al., 2004; Bartolotta et al., 2005). For these reasons, ultrasonographic imaging with contrast enhancement provides a reliable measurement vascularity of tumors.

In this study, we detect tumor vascularity macroscopically in PTC tumors with CEUS. Compared with the peripheral parenchyma, 60 PTC nodules showed low heterogeneous enhancement and 2 nodules showed slightly high enhancement, and the PI of CEUS in PTC was lower than that of in peripheral parenchyma. In addition, the highest of blood perfusion of PTC was lower than that of peripheral parenchyma. Moreover, the microvessels of 62 PTC nodules were also less than that of peripheral parenchyma. The "slow or slightly high enhancement" phenomenon might be attributable to the comparatively small diameters (mean, $8.3 \pm 3.7 \mathrm{~mm}$ ) of the PTC nodules in our study group. This result was consistent with report of Bartolitta (BartolottaMidiri et al., 2006). The report of Bartolitta showed that the enhancement of thyroid nodule was associated with the diameter of nodule. The diameter of thyroid nodules measuring less than $1 \mathrm{~cm}$ showed mainly absent vascularization, those between 1 and $2 \mathrm{~cm}$ revealed faint dotted contrast enhancement and, finally, nodules with a diameter larger than $2 \mathrm{~cm}$ presented diffuse contrast enhancement (BartolottaMidiri et al., 2006).

There have been other studies about angiogenetic evaluation of malignant tumors by CEUS. CEUS imaging had been applied to distinguish benign from malignant thyroid nodules or breast masses due to the microvascular imaging function. Sedelaar et al (2001) reported that 3-dimensional power Doppler CEUS could be applied to visualize lesions with increased MVD in prostate cancer. Zhili Wang et al confirmed that the PI in CEUS could reflect the MVD and be helpful for evaluation of tumor vascularity in Hepatocellular Carcinoma (WangTang et al., 2007). In the study of D'Onofrio, the relationship between tumor vessels and CEUS in pancreatic tumors using SonoVue and enhanced CT scans was compared, and it was indicated that the correlation between CEUS 
and tumor vessels was much better than that of enhanced $\mathrm{CT}$ and tumor vessels. Xi Wei et al found a significant relationship between CEUS feature and MVD counts in thyroid cancer in women with breast cancer, and CEUS can facilitated the diagnosis of thyroid cancer (Wei et al., 2013). Immunohistochemical results showed that the MVD of PTC was significantly higher than that of peripheral tissue. On CEUS, the PI of PTC was significantly correlated with MVD. Thus, our results showed that tumor vascularity in PTC could be evaluated by CEUS.

In this study, quantitative TomTec software was applied. It made the choice of the ROI freely and observation of the enhancement pattern of the contrast agent in the ROI in real time. Also, the quantitative feature of microcirculation perfusion in the ROI could be calculated easily and accurately. In addition, it can provide motion compensation and evaluation of contrast agent perfusion in the ROI objectively.

In conclusion, PI on CEUS can be used for evaluating PTC tumor vessels and can provide a noninvasive feature for clinical evaluation of tumor vascularity. No intra- or inter-rater assessment as well as the repeatability or reliability of the technique was included in this study. Further study should be conducted to determine whether CEUS can depict tumor vascularity changes caused by antiangiogenic treatments and the correlation between CEUS and PTC prognosis and metastasis.

\section{Acknowledgement}

The author (s) declare that they have no competing interests.

\section{References}

Accurso A, Rocco N, Palumbo A, Leone F (2005). Usefulness of ultrasound-guided fine-needle aspiration cytology in the diagnosis of non-palpable small thyroid nodules. Tumori, 91, 355 .

Asioli S, Erickson LA, Sebo TJ, et al (2010). Papillary thyroid carcinoma with prominent hobnail features: a new aggressive variant of moderately differentiated papillary carcinoma. A clinicopathologic, immunohistochemical, and molecular study of eight cases. Am J Surg Pathol, 34, 44-52.

Bartolotta TV, Midiri M, Galia M, et al (2006). Qualitative and quantitative evaluation of solitary thyroid nodules with contrast-enhanced ultrasound: initial results. Eur Radiol, 16, 2234-41.

Bartolotta TV, Midiri M, Quaia E, et al (2005). Liver haemangiomas undetermined at grey-scale ultrasound: contrast-enhancement patterns with SonoVue and pulseinversion US. Eur Radiol, 15, 685-93.

Brander A, Viikinkoski P, Nickels J , Kivisaari L (1991). Thyroid gland: US screening in a random adult population. Radiology, 181, 683-7.

Du J, Li F-H, Fang H, et al (2008). Correlation of real-time gray scale contrast-enhanced ultrasonography with microvessel density and vascular endothelial growth factor expression for assessment of angiogenesis in breast lesions. J Ultrasound Med, 27, 821-31.

Halpern EJ, Ramey JR, Strup SE, et al (2005). Detection of prostate carcinoma with contrast-enhanced sonography using intermittent harmonic imaging. Cancer, 104, 2373-83. Hayashi K, Tozaki M, Sugisaki M, et al (2002). Dynamic Multislice Helical CT of ameloblastoma and odontogenic keratocyst: correlation between contrast enhancement and angiogenesis. J Comput Assist Tomogr, 26, 922-6.

Kabakci N, Igci E, Secil M, et al (2005). Echo contrastenhanced power doppler ultrasonography for assessment of angiogenesis in renal cell carcinoma. J Ultrasound Med, 24, 747-53.

Liotta LA, Kleinerman J, Saidel G M (1974). Quantitative relationships of intravascular tumor cells, tumor vessels, and pulmonary metastases following tumor implantation. Cancer Res, 34, 997-1004.

Quaia E, Calliada F, Bertolotto M, et al (2004). Characterization of focal liver lesions with contrast-specific us modes and a sulfur hexafluoride-filled microbubble contrast agent: diagnostic performance and confidence1. Radiology, 232, 420-30.

Santacroce L, Gagliardi S, Scott Kennedy A (2009). Thyroid, papillary carcinoma. Carcinomas of Endocrine Organs (emedicine from WebMD).

Schlemmer H-P, Merkle J, Grobholz R, et al (2004). Can preoperative contrast-enhanced dynamic MR imaging for prostate cancer predict microvessel density in prostatectomy specimens? Eur Radiol, 14, 309-17.

Sedelaar JM, van Leenders GJ, Hulsbergen-van de Kaa C A, et al (2001). Microvessel density: correlation between contrast ultrasonography and histology of prostate cancer. Eur Urol, 40, 285-93.

Solbiati L, Ierace T, Tonolini M , Cova L (2004). Guidance and monitoring of radiofrequency liver tumor ablation with contrast-enhanced ultrasound. Eur J Radiol, 51, 19-23.

Tamai H, Takiguchi Y, Oka M, et al (2005). Contrast-enhanced ultrasonography in the diagnosis of solid renal tumors. $J$ Ultrasound Med, 24, 1635-40.

Wang B, Gao Z, Yan X (2005). Correlative study of angiogenesis and dynamic contrast-enhanced magnetic resonance imaging features of hepatocellular carcinoma. Acta Radiologica, 46, 353-8.

Wang S-F, Zhao W-H, Wang W-B, et al (2013). Clinical features and prognosis of patients with benign thyroid disease accompanied by an incidental papillary carcinoma. Asian Pac J Cancer Prev, 14, 707-11.

Wang Z, Tang J, An L, et al (2007). Contrast-enhanced ultrasonography for assessment of tumor vascularity in hepatocellular carcinoma. J Ultrasound Med, 26, 757-62.

Wei X, Li Y,Zhang S, et al (2013). Evaluation of microcirculation of thyroid cancer in Chinese females with breast cancer using contrast-enhanced ultrasound (CEUS) combined with VEGF and microvessel density (MVD). Clin Hemorheol Microcirc.

Weidner N (1998). Tumoural vascularity as a prognostic factor in cancer patients: the evidence continues to grow.J Pathol, 184, 119-22.

Wu H-X, Zhang B-J, Wang J, et al (2013). Conventional ultrasonography and real time ultrasound elastography in the differential diagnosis of degenerating cystic thyroid nodules mimicking malignancy and papillary thyroid carcinomas. Asian Pac J Cancer Prev, 14, 935-40.

Yang WT, Gary M, Lam PK, et al (2002). Correlation between color power doppler sonographic measurement of breast tumor vasculature and immunohistochemical analysis of microvessel density for the quantitation of angiogenesis. $J$ Ultrasound Med, 21, 1227-35.

Zhuang H, Yang Z, Chen H, et al (2012). Time-intensity curve parameters in colorectal tumours measured using double contrast-enhanced ultrasound: correlations with tumour angiogenesis. Colorectal Disease, 14, 181-7. 